\title{
Experimental Model-Based Linearization of a S.I. Engine Gas Injector Flow Chart
}

\author{
Emiliano Pipitone* - Stefano Beccari - Marco Cammalleri - Giuseppe Genchi \\ University of Palermo, Italy
}

\begin{abstract}
Experimental tests previously executed by the authors on the simultaneous combustion of gasoline and gaseous fuel in a spark ignition engine revealed the presence of strong nonlinearities in the lower part of the gas injector flow chart. These nonlinearities arise via the injector outflow area variation caused by the needle impacts and bounces during the transient phenomena that take place in the opening and closing phases of the injector and may seriously compromise the air-fuel mixture quality control for the lower injection times, thus increasing both fuel consumption and pollutant emissions.

Despite the extensive literature about the operation and modelling of fuel injectors, there are no known studies focused on the nonlinearities of the gas injector flow chart and on the way they can be reduced or eliminated. The authors thus developed a mathematical model for the prediction of mass injected by a spark ignition (S.I.) engine gas injector, validated through experimental data. The gas injector has been studied with particular reference to the complex needle motion during the opening and closing phases, which may strongly affect the amount of fuel injected.

In this work, the mathematical model previously developed has been employed to study and determine an appropriate injection strategy in order to linearize the injector flow chart to the greatest degree possible. The injection strategy proposed by the authors is based on minimum injection energy considerations and may be easily implemented in current engine control units (ECU) without any hardware modification or additional costs. Once calibrated by means of simulation, this strategy has been validated by experimental data acquired on an appropriately equipped injector test bench. As a result, the real injector flow chart has been substantially improved, reducing its deviation from linearity to one third of the original flow chart, which is an excellent result, especially if the typical measurement dispersion of the injected mass is taken into account. The injection strategy proposed by the authors could extend the linear behaviour of gas injectors and improve the fuel supply by means of a simple software update of the ECU, thus obtaining higher engine efficiency and lower pollutant emissions.
\end{abstract}

Keywords: fuel injector, injection strategy, spark ignition engine, modelling and optimization

\section{O INTRODUCTION}

Since the introduction of polluting emission regulations for passenger cars, fuel injection systems have become an indispensable part of modern spark ignition engines, due to their capability to accurately meter the mass of fuel employed at each engine cycle.

A typical gaseous fuel (liquefied petroleum gas, LPG, or compressed natural gas, CNG) multi-point injection system for spark ignition (S.I.) engines is composed of the elements reported in Fig. 1: the regulator reduces the gas pressure from the high level in the tank (where LPG is stored at around 10 bar and $\mathrm{CNG}$ at around $200 \mathrm{bar}$ ) to the low level in the fuel rail (about 2 bar for LPG and 10 bar for CNG); hence, when the injector is activated, the fuel arrives to the inlet duct (port injection) of the engine; the flow through the gas injector can be assumed to be equivalent to the flow through a convergent nozzle: in a chocked flow condition (i.e. supposing that, as usual, the ratio between fuel rail pressure and manifold pressure is $\geq 2$ ), the gas flow depends only on pressure and temperature upstream from the injector; this makes the injected mass directly proportional to the "injection time" (i.e. the duration of the time interval during which the injector is activated), regardless of the pressure level in the intake manifold of the engine. This proportionality makes the injector characteristic almost linear on the injector flow chart, which is the diagram used to represent the amount of fuel injected for any injection time. An electronic control unit (ECU) adjusts the injected fuel mass, and then the airfuel ratio, acting on the injection time, whose values are stored in memory, by means of proper tables, as a function of engine speed and load. In some operating conditions, typically lower engine loads, this openloop control is integrated by means of a more accurate closed-loop control, which, using the lambda sensor output signal, performs continuous adjustment on the amount of fuel injected to maintain air-fuel ratio at the stoichiometric value, thus minimizing pollutant emissions.

This simple closed-loop control, based on a single feedback parameter (e.g. lambda sensor output signal), can be performed due to the linearity of the injector characteristic.

Previous experimental tests carried out by the authors of this paper showed, however, the existence of strong nonlinearities in the lower part of the gas injector flow chart. These nonlinearities may compromise the air-fuel ratio control performed by the engine ECU, causing unstable corrections of the 
injected fuel mass, thus leading to both poor fuel economy and high pollutant emissions (spark ignition engine catalytic converters has a very low efficiency for non-stoichiometric mixtures).

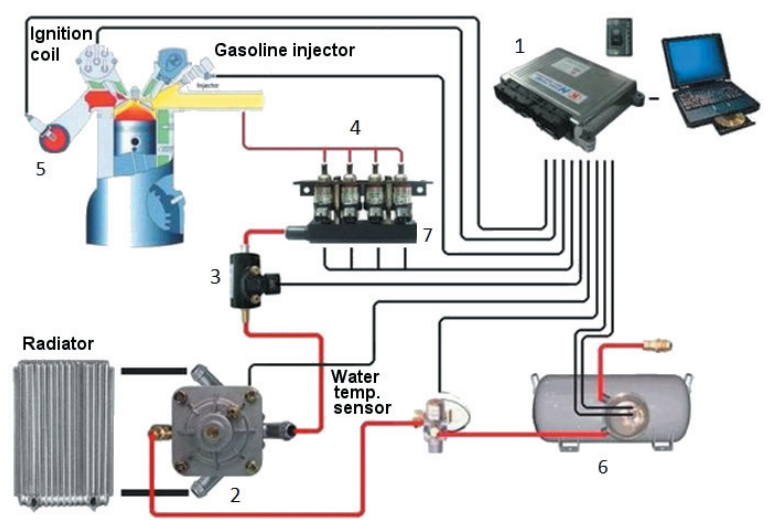

Fig. 1. Gaseous fuel multi point injection setup: 1) ECU, 2) pressure regulator, 3) filter valve, 4) gas injectors, 5) lambda sensor, 6) gas tank, and 7) fuel rail

With the aim of studying and reproducing the nonlinear behaviour of the gas injector, the authors created a mathematical model [1] for the evaluation of the complex needle motion during the entire injection event, with maximum accuracy on the opening and closing phases, which have been recognized to be determinant in the generation of the nonlinearities.

In the present work, the authors employed the developed model to study and propose a suitable injection strategy with the aim of suppressing the nonlinear behaviour of an actual gas injector, thus linearizing its flow chart and extending its range of utilization towards the lower injection times.

The main advantage of the determined injection strategy is its ease of implementation in current production engines, since a simple ECU software update is required.

\section{LITERATURE OVERVIEW}

An extensive literature is currently available on the simulation and modelling of internal combustion engine injection systems. Compression ignition (C.I.) engines typically have high pressure (1600 to 2000 bar) common rail injectors which, activated by a solenoid or by a piezoelectric element, use the high fuel pressure to move the needle and open the nozzle. Spark ignition engines may be port injected or direct injected: in the first case, low pressure (3 to 10 bar, depending on fuel type) injectors are usually employed, while in the second case significantly higher injection pressure may be involved (100 to
500 bar). Despite the extensive available literature on injection system simulation, very few works cover the dynamic modelling of the injector needle motion, which is the focus of this paper.

With regard to common rail injection systems, the needle motion has been dealt with extensively in literature: for example, the fluid-dynamic model presented in [2] allows predicting the injection pressure variations and deriving control laws for the rail pressure controller, while in [3] the model developed using a commercial code also predicts needle lift and injection rate for different injection pressures; the common rail piezoelectric injector model realized in [4] takes into account both the hydraulic part (fluid flow, discharge coefficients) and the mechanical part (needle movement, seats elastic deformation) with the aim of predicting different flow rate profiles.

With regard to gasoline direct injection, the model developed in [5] refers to a piezoelectric injector and compares the capability of lumped parameters and distributed parameters to describe the needle motion and the behaviour of piezoelectric elements.

On account of the dumping effect of liquid fuels, which completely suppress any needle bounces, none of the abovementioned works [2] to [5], however, report any injector flow chart nonlinearities. The problem is a feature of gas injection systems.

The modelling of gas injection in S.I. engines is handled in [6], where the details of fuel spray formation and mixture with air are explored, while the dynamic behaviour of the injector needle is discussed in [7], where different model predictive control (MPC) schemes are presented for the control of an electromagnetically actuated mass-spring-damper system for automotive applications: in this work, however, the solenoid power voltage is assumed to vary between zero and $350 \mathrm{~V}$ while, in the present paper, the authors considered a constant power voltage of $13 \mathrm{~V}$, which complies with the actual automotive electric system specifications.

The natural gas injection system modelled in [8] presents control strategies for the optimization of the injection system operation focusing on the fluid-dynamic behaviour of the whole injection system (fuel rail, pressure control valve, injectors). Even if focusing on gas injections, however, none of the abovementioned works [6] to [8] deals with the nonlinearities produced by the needle bounces during the opening and closing phases of the injector. Only the work presented in [9] focuses on the suppression of gas injectors needle bounces, even if with an entirely different purpose, i.e. the prevention of fatigue stress 
damages. Moreover, contrary to the approach followed in the present paper, the implementation of the method proposed in [9] requires a substantial modification of the injector power supply system.

The presence of a nonlinear zone in the injector flow chart, however, has never been studied in detail, least of all its correlation with the needle motion. This consideration led the authors to develop a proper mathematical dynamic model of the gas injector and to study a proper injection strategy with the aim to linearize the injector flow chart: this would allow improving the air-fuel mixture quality control while minimizing both fuel consumption and polluting emissions.

\section{SOLENOID INJECTOR DYNAMICS}

Fig. 2 reports a cutaway of the solenoid gas injector [10] used in the test, while Fig. 3 shows a typical electrical circuit used to energize the injector solenoid; this circuit is composed of the power supply, the injector solenoid and the power transistor activated by TTL pulses, which may be generated by the engine ECU or by a personal computer.

The injector is mainly composed of a mechanical part (the needle) and an electric part (the solenoid), and these two parts interact, influencing each other through the electromagnetic field. The needle movement influences the solenoid current, which in turn, acts on the needle by the electromagnetic force.

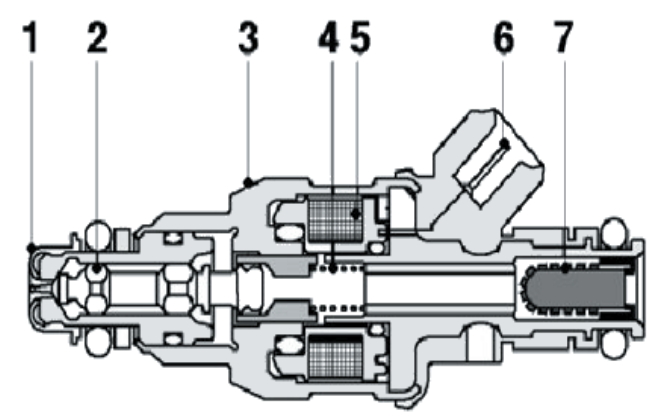

Fig. 2. Cutaway of the fuel injector used in the test: 1) pintle, 2) needle, 3) armature, 4) spring, 5) solenoid winding, 6) electrical terminals, 7) fuel strainer

When the solenoid is not energized (i.e. the electrical circuit is open), the needle is kept in closing position by both the fuel pressure and the spring load. When the ECU activates the transistor (which can be considered a "digital switch"), this closes the electrical circuit, and the current rises in the solenoid windings (see Fig. 3), according to the R-L circuit law; the needle is moved by the electromagnetic force from the closed towards the open position, thus knocking against the stopping surface at the end of the lift. Here, the needle bounces and moves towards the closed position, where another impact may occur. Under the action of the electromagnetic field, the needle will, however, be pushed toward the open position, thus producing other bounces. If the injection time is long enough, the needle will conclude all the bounces and then, compelled by the electromagnetic field, remain in the open position.

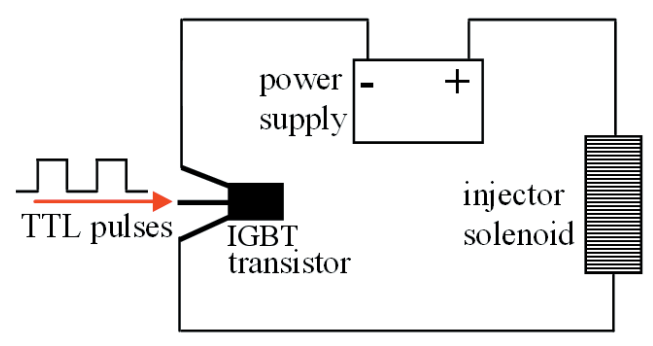

Fig. 3. Schematic representation of the usual electrical circuit involved in injector operation

Once finished the injection time, the ECU deactivates the transistor that opens the circuit, producing an instantaneous drop of the solenoid current; the needle is then forced to return to the closed position by the fuel pressure and the spring load, thus knocking against the closed position seat and producing other bounces. Figs. 4 and 5 show the output signal from an accelerometer mounted on the armature of the injector used for test, during the injector opening and closing phases.

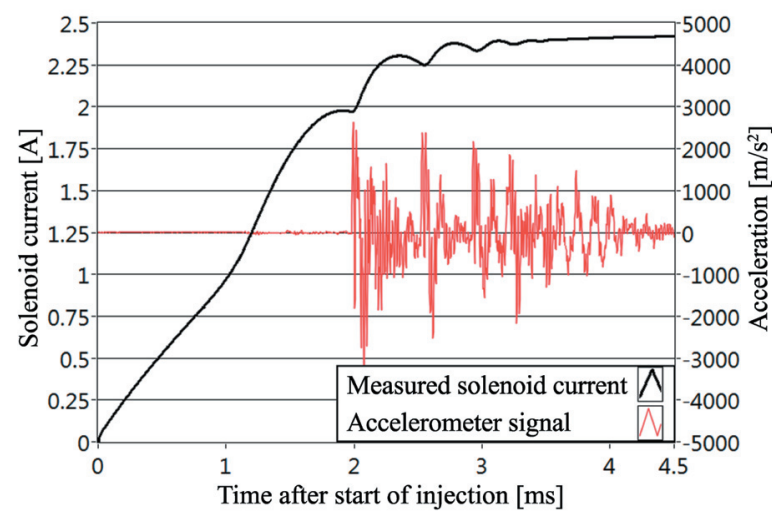

Fig. 4. Waveforms of solenoid current and armature acceleration during injector opening phase

As can be observed, the substantial impacts that occur both in the opening and closing phases cause prominent spikes on the accelerometer output signal. 
The same diagrams also show that the measured solenoid current, which, during the opening phase, is characterized by the presence of several cusps: due to the reciprocal interaction between needle movements and coil-winding current [1] and [9], the abrupt velocity variation during an impact causes a rapid change in the current first derivative. These current cusps are not present in the closing transient since, after the end of the injection, the electric circuit is open, and the solenoid current is null.

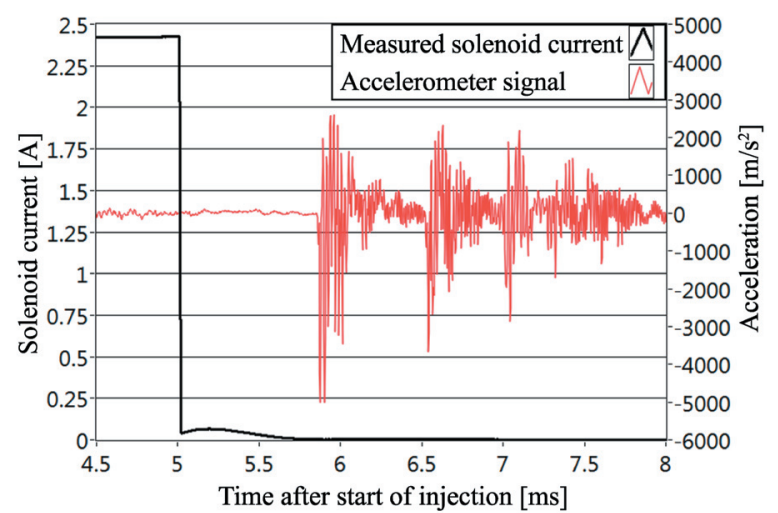

Fig. 5. Waveforms of solenoid current and armature acceleration during injector closing phase

Fig. 4 also shows that, for the tested injector fed with air at 10 bar, in the opening phase, the bounces continue for about $4 \mathrm{~ms}$, while in the closing phase (see Fig. 5) their duration is shorter, i.e. about 3 ms. The importance of these bounces relies on the significant variations they produce on the injected mass, since the instantaneous flow section depends on the needle position; therefore, assuming a linear correlation between the flow section area and needle position, it results that the injected mass depends on the value reached by the integral of the needle position over time. Moreover, when the injection time is below the opening phase transient duration $(\approx 4 \mathrm{~ms}$ for the injector tested fed with air at 10 bar), not only is the needle transient not completed, but it is also influenced by the duration of the injection itself; the impact energy of the needle on the opening stop surface, in effect, depends on its kinetic energy, which, in turn, is related to the duration of the electromagnetic force applied, and hence to the injection time. It results hence that, for injection times shorter than $4 \mathrm{~ms}$, changing the injection duration modifies the needle movement and hence the integral of its position, which causes a variation in the injected mass. This introduces a non-linear dependence between the injected mass and the injection time, as evident in the injector flow chart shown in Fig. 6.

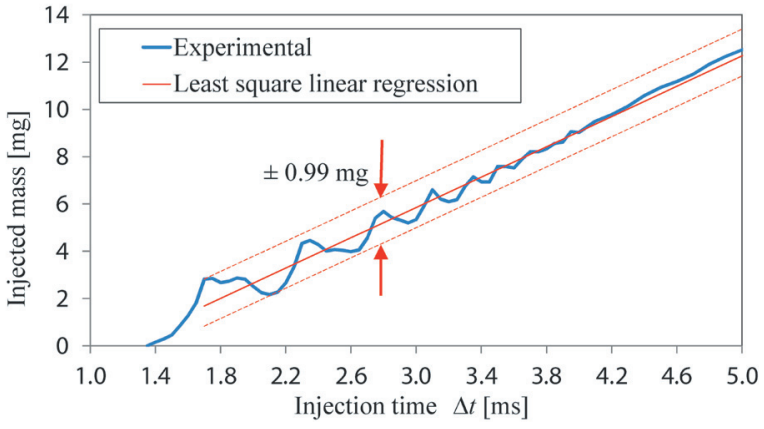

Fig. 6. Experimental injector flow chart obtained with air at 10 bar

This diagram reports the measured injected mass for each of the injection time imposed to the injector, fed with air at 10 bar. It is noteworthy that this diagram does not represent the integral of the gas mass flow as a function of time, but rather the measured total injected mass at the end of each single injection, whose duration is the injection time $\Delta t$. As can be noted, for injection durations shorter than the bounces duration $(\approx 4 \mathrm{~ms})$, the needle bounces have a considerable influence on the total injected mass, causing the presence of strong nonlinearities, which may seriously compromise the injected mass control; the ideal flow chart is represented by a straight line, suitable for a pure linear control of air-fuel ratio; therefore, the deviation from the ordinary least square (OLS) line can be considered a measure of the injected mass control quality: the higher the deviation is, the worse the control is. As can be seen in Fig. 6, for the injector flow chart obtained with air at 10 bar, this deviation amounts to almost $\pm 1 \mathrm{mg}$, which means that using the OLS line instead of the experimental data, the fuel mass control would be subjected to a maximum error of $1 \mathrm{mg}$, which, depending on the amount of gas to inject, can cause a very large error.

The strong nonlinearities of such a flow chart arise from the flow section variations caused by the needle bounces [1], whose intensities, as already pointed out, are related to the needle's kinetic energy, which depends on the duration of the electromagnetic force applied. During the opening phase, due to the bounces on the two stopping surfaces, the needle frequently reverses its motion, while the electromagnetic force always acts in the same direction: this implies that, depending on the needle velocity, the electromagnetic thrust may accelerate or slow down the needle, thus changing its effect in terms of the needle's kinetic energy and, in turn, in terms of integral of the needle position, which is proportional to the injected mass. On account of this, it can be understood that, during the opening phase, increasing the injection time may 
have opposite effects on the injected mass, depending on the needle position and velocity. This conclusion has been confirmed by experimental observation, performed using a $100 \mathrm{MHz}$ oscilloscope, of the solenoid current and armature acceleration waveforms together with mass flow data acquisition carried out for air at 9 bar and injection times between 1.8 and $2.5 \mathrm{~ms}[1]$.

When the injection time is long enough to let the needle complete all the opening bounces (i.e. $\geq 4 \mathrm{~ms}$ for 10 bar air pressure), the complete opening and closing transient phenomena repeat at each single injection and thus have no effect on the total injected mass, which then becomes a linear function of the injection time, as observable in Fig. 6.

The nonlinearities of the injector flow chart can cause inaccurate control over the engine's air-fuel ratio; this can lead to both higher fuel consumption and higher pollutant emissions, also due to the low efficiency of the catalytic converter for nonstoichiometric air-fuel mixtures. These nonlinearities have not been observed using gasoline: therefore, this study focuses on gaseous fuel injector dynamics.

It could be argued that the problem could be overcome by properly selecting the gas injector, so as to always let it operate on its linear range, thus making the nonlinearities an unused part of the flow chart; unfortunately, this is not always possible, since maximum injection time must respect limits imposed by the available time at the maximum engine speed; moreover, the "ideal" injector may not be available from the manufacturer, or may not be economically favourable; this may lead to the installation of a gas injector that, for the particular engine, operates in the nonlinear part of its diagram. For example, the injector used in this study is part of the CNG injection system of a series production bifuel engine from FIAT; data acquired on the engine test bench revealed a maximum injection time of 8.8 $\mathrm{ms}$, which corresponds to the injection of the full load fuel mass; in contrast, the injector flow chart revealed the nonlinear range to lie between injection times of 1.8 and $3.5 \mathrm{~ms}$ : experimental data show that when the injector exits the nonlinear range (i.e. with injection time of $3.5 \mathrm{~ms}$ ), the injected mass is between 18 and $36 \%$ of the full load mass (depending on engine speed), and the engine torque is between 10 and $23 \%$ of the maximum, which, as example, could be a typical condition in urban areas. In this case the characteristics of the selected injector do not allow operating exclusively in the linear range. A very accurate (and time-consuming) calibration of the ECU injection map, however, may attenuate the effects of the nonlinearities, thus allowing the engine to comply with current pollutant emission limits and regulations.

Other particular engine applications may however involve injector operations in the nonlinear range, such as supercharged engines or Double-Fuel combustion. In a supercharged engine, basically, a compressor is used to increase the air pressure in the manifold, thus letting the engine to draw a greater amount of air-fuel mixture and produce higher power; this obviously implies higher fuel flows, and thus longer injection times, which, may compel the adoption of larger injectors; for the lower engine loads (e.g. urban operative condition), the same injectors could thus be operated with injection times so short that they enter the nonlinear range.

The second case refers instead to the simultaneous combustion of gasoline and gaseous fuels, such as LPG or CNG, which has been successfully tested by the authors [11], and [12] and by other research groups [13] to [15]; this kind of combustion can be easily implemented in bi-fuel engines as a third operative mode realized by the injections of both gasoline and gas within the same engine cycle, requiring the adoption of shorter gas injection times (even 20\% of nominal values): this may induce the gas injector to operate in the nonlinear range, thus causing poor air-fuel ratio control, with consequent increases in pollutant emissions and decreases in engine efficiency.

The injection strategy proposed could be effectively employed to linearize the injector flow chart and allow a better control of the engine air-fuel ratio on a wider part of the injector flow chart. The approach followed in the present paper relies on the use of numerical simulations for the definition of a proper injection strategy capable of avoiding needle bounces; the optimal injection strategy determined has been implemented on a real injector test bench and experimentally optimized, thus removing most of the unwanted nonlinearities from the real injector flow chart.

\section{SIMULATIONS}

As already mentioned, in previous works [1] and [16] the authors realized a mathematical model for the simulation of the complex needle motion during the opening and closing phases of a gas injector, in order to predict the amount of fuel injected for each injection time; the model, whose main equations and structure are briefly resumed in Appendix B, has been calibrated by means of the experimental data obtained on a proper test bench using a natural gas injector fed with air at 9 bar, and successfully validated by means 
of the experimental data obtained injecting air at 8 and 10 bar. Fig. 7 shows a comparison between measured and simulated injector flow charts relative to the injection of air at 10 bar. The results explored in this paper, both from simulations and from experimental tests, all refer to the injection of air at 10 bar absolute pressure.

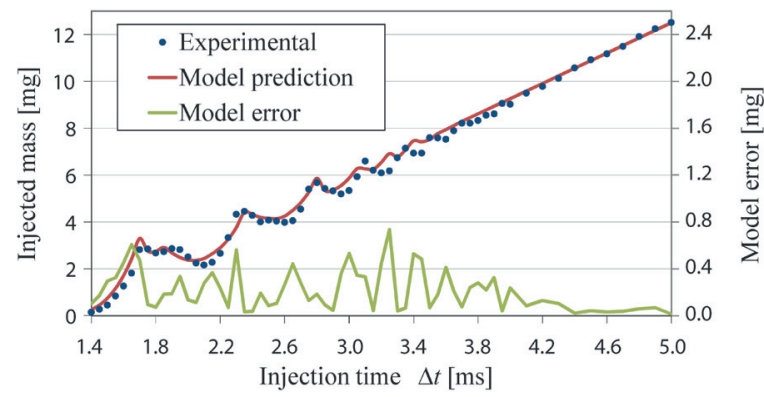

Fig. 7. Comparison between measured and computed injected mass

As shown, a very good fit has been obtained between experimental data and model prediction, since the nonlinearities of the experimental diagram are accurately replicated by the model. Fig. 7 also shows the error (i.e. difference between calculated and measured injected mass) distribution, whose mean and maximum values resulted in 0.22 and $0.74 \mathrm{mg}$, respectively. In all the cases, the model evaluation accuracy resulted in being comparable to the test measurement uncertainties [1], which are not related to mass flow measurement errors (always less than $1 \%$ ) but rather to the typical injected mass measurement dispersion around the mean values (as reported in Fig. 19).

A further confirmation of the model's predictive capacity has been obtained by the comparison between the measured and the evaluated solenoid current in the same condition of air pressure and injection time; for example, the diagram of Fig. 8 shows the good agreement between the experimental and the numerical current during the injector opening phase; the first cusp is due to variation of the steel magnetic permeability, while the other cusps are connected to the sudden speed change of the needle due to the impacts on the seat surface.

Fig. 9 shows a typical model output, i.e. the solenoid current and the needle displacement as a function of time: specifically, the diagram refers to a $5 \mathrm{~ms}$ injection of air at 10 bar. The opening phase bounces are evident both in the needle displacement and in the solenoid current, and their duration is about $3.6 \mathrm{~ms}$; consequently, this is also the minimum injection time of the linear part in the simulated flow chart of Fig. 7.

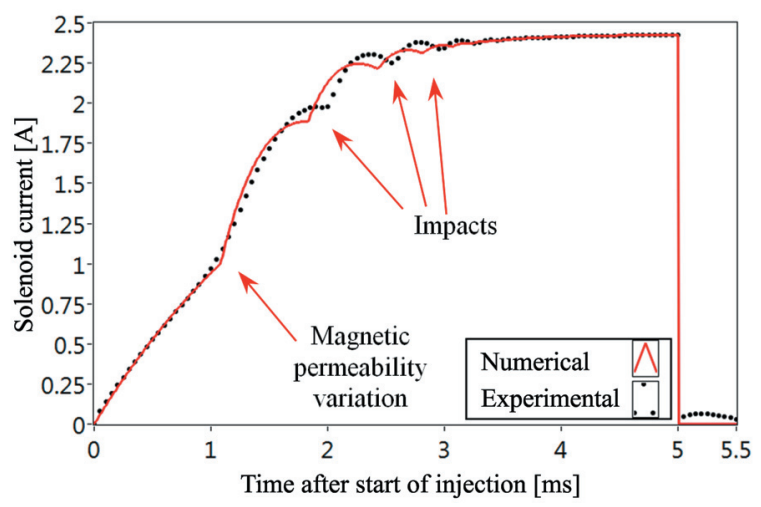

Fig. 8. Measured and simulated solenoid current

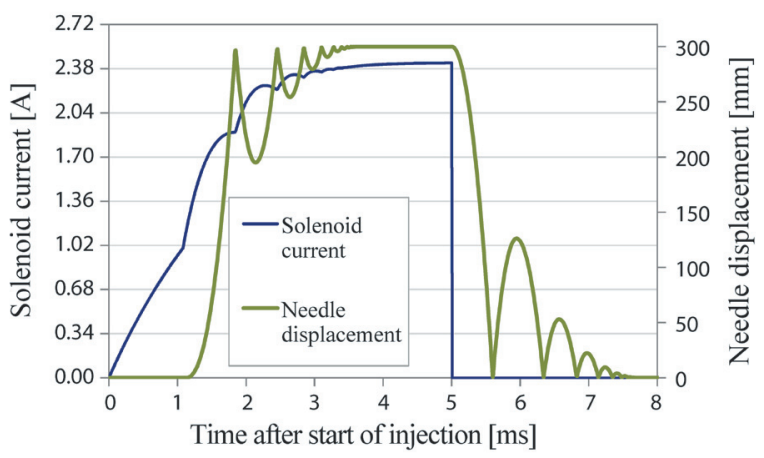

Fig. 9. Simulated solenoid current and needle displacement $(\Delta t=5 \mathrm{~ms})$

The closing phase bounces have a duration of about $2.5 \mathrm{~ms}$ and are evident only in the displacement waveform, since, at the end of the injection, the transistor (see Fig. 3) is deactivated and this opens the electric circuit causing the current to immediately fall down to zero.

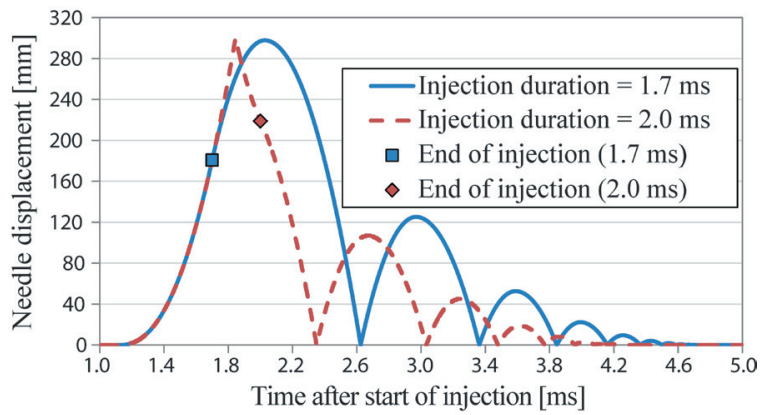

Fig. 10. Simulated needle displacement for two injections with $\Delta t=1.7$ and $2.0 \mathrm{~ms}$

Fig. 10 shows the needle displacement evaluated by the model for two different injection durations, i.e. 
1.7 and $2.0 \mathrm{~ms}$, with air at $10 \mathrm{bar}$. In the case of the 2 $\mathrm{ms}$ injection, the electromagnetic force still acts after the first impact, thus slowing down the needle, whose successive impacts have lower energy and thus cause smaller bounces. As a result, the $2.0 \mathrm{~ms}$ injection is characterized by a lower value of the integral of the needle position, which, as already mentioned, means a lower value of the integral of the mass flow, and hence, a lower injected mass; the simulated injector flow chart in Fig. 7 confirms that the $1.7 \mathrm{~ms}$ injection gives a higher injected mass than the $2.0 \mathrm{~ms}$ injection.

\section{OPTIMAL INJECTION STRATEGY}

The needle bounces on the stopping surfaces originate from the excess of kinetic energy acquired by the needle during the opening lift, which in turn is due to the excess energy transferred through the electromagnetic field, and hence by the solenoid. It can be easily understood that in order to avoid any bounce, the needle should arrive at the opening stop surface with no kinetic energy, and be maintained in this position by the electromagnetic thrust. This could be pursued by a proper modulation of the solenoid current in order to progressively reduce the electromagnetic thrust on the needle during the lift, thus involving the minimum energy necessary to shift the needle from the closed to the open position. The entire excess of energy transferred to the needle, with respect to the minimum required, is completely dissipated during the bounces by the mechanical friction between the needle and guides, by the gas viscous forces and by the energy loss at each impact: the more the needle moves or impacts, the more energy it dissipates.

The modulation of the solenoid current would however require the modulation of the voltage supplied to the injector solenoid, which instead, as typical in automotive engines, is constant and equal to the battery voltage. Given the difficulty of operating with a variable solenoid voltage supply, the energy transferred to the needle during the opening lift can be elsewhere modulated by acting on the duration of the injection pulse: this can be then divided in half, with the first part dedicated to shifting the needle from the closed to the open position without bounces, and the second part dedicated to maintaining the needle in the open position and let the fuel flow. The authors thus focused on this division, which can be realized by the simple interruption of the injection pulse, characterized by two parameters: the interruption delay $\delta$ with respect to the start of injection and the duration $\tau$, both indicated in Fig. 11. It is noteworthy that, in a conventional way, the injection time has been always considered the time interval between the first rising front and the last falling front of the injection pulse, as shown in Fig. 11.

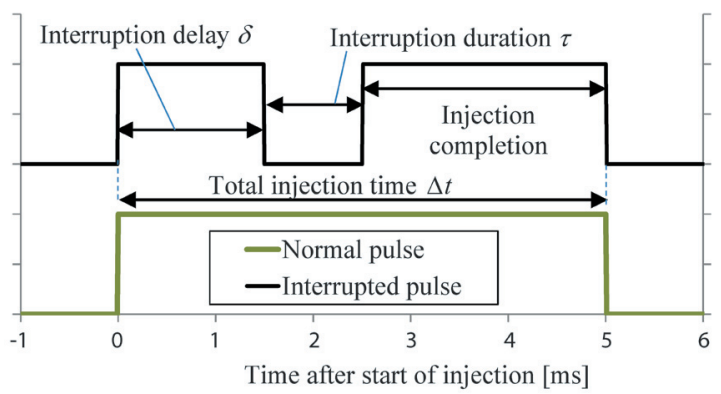

Fig. 11. Injection pulse with and without interruption

The authors used the mathematical model previously developed to determine the two parameters values, which allow avoiding needle bounces, so as to linearize as much as possible the injector flow chart. Before the research for the optimal interruption parameters was started, the model was further improved in order to adequately take into account a phenomenon not revealed by the first experimental campaign. As reported in Fig. 12, the solenoid current measured during an interrupted injection shows a sort of extra-current that substantially modifies the current waveform (and hence the needle motion) and is due to the partial discharge of the energy accumulated by the solenoid that occurs during the injection interruption. Details on this phenomenon and on the model modifications introduced by the authors are given in Appendix A.

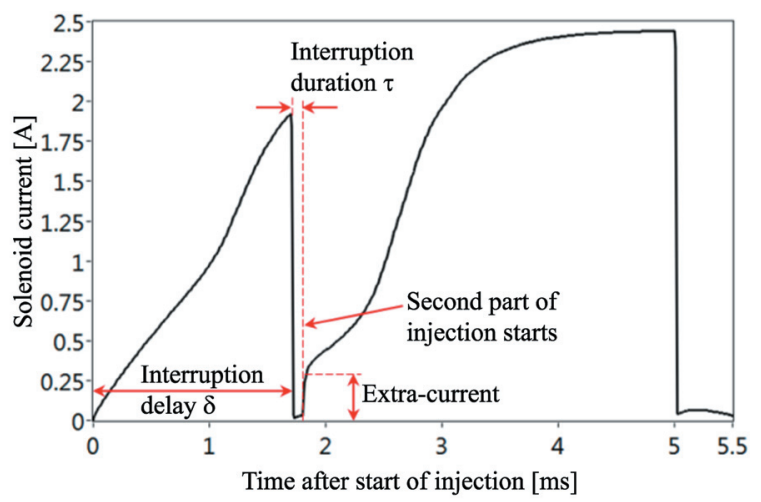

Fig. 12. Measured solenoid current for an interrupted injection pulse (injection time $=5 \mathrm{~ms}$ )

Once refined, the model was employed to perform several simulations with the aim of determining the optimal values to assign to the interruption parameters $\delta$ and $\tau$ in order to avoid any needle bounce. According 
to what was already explained above, this condition should also minimize the energy $E$ employed for the needle shift from the closed to the open position; the authors thus adopted the energy transferred to the needle in the opening phase as objective function $\phi$ of the search algorithm:

$$
\varphi=E=V \cdot \int_{0}^{t^{*}} i \cdot d t,
$$

being $t^{*}$ the time necessary to let the needle complete all the opening phase bounces and stops in the open position (i.e. the opening phase duration), $V$ the constant supply voltage and $i$ the solenoid current.

As a first step, the authors considered the injection time of $5 \mathrm{~ms}$, which, as reported in Fig. 9, gives rise to several bounces in the opening phase transient. A quite simple search algorithm has been employed, since an entire matrix of interruption delay $\delta$ (ranging from 1.55 to $1.76 \mathrm{~ms}$ with steps of 0.003 $\mathrm{ms}$ ) and duration $\tau$ (ranging from 0.01 to $0.15 \mathrm{~ms}$ with steps of $0.002 \mathrm{~ms}$ ) has been tested using the model, evaluating the objective function $\phi$ on the basis of the resulting simulation output. This procedure allowed tracing the $\phi$ surface, shown in Fig. 13 as a function of the two variables delay $\delta$ and duration $\tau$; as can be noted, the absolute minimum region is visible, whose coordinates represent hence the best (minimum energy) values of interruption delay and duration. This is also shown in Fig. 14, which shows a contour plot of the $\phi$ surface; the best interruption parameters are $\delta=1.64 \mathrm{~ms}, \tau=0.038 \mathrm{~ms}$.

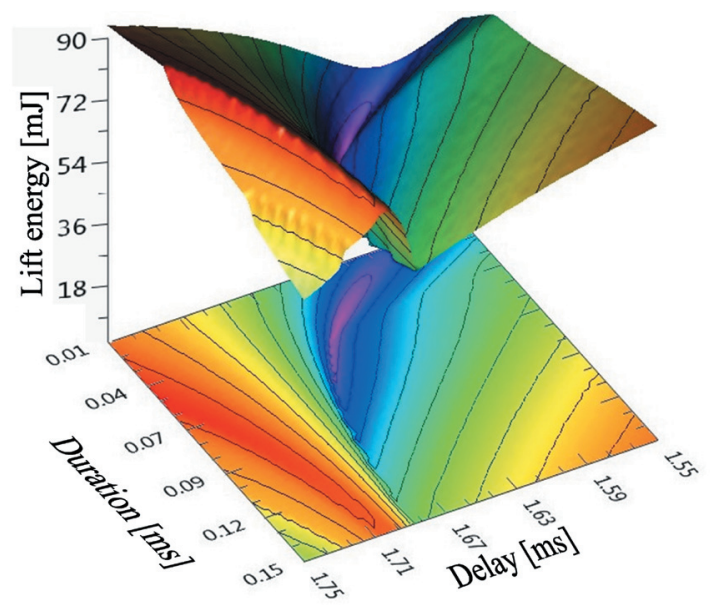

Fig. 13. Surface of the opening phase energy as a function of the two interruption parameters

In conclusion, for the injector tested and fed with air at $10 \mathrm{bar}$, the optimal injection pulse interruption has a very short duration and should be placed before the first impact to occur. The effects of these optimal interruption parameters on the $5 \mathrm{~ms}$ injection of air at 10 bar are reported in Fig. 15 in terms of both the solenoid current and needle displacement.

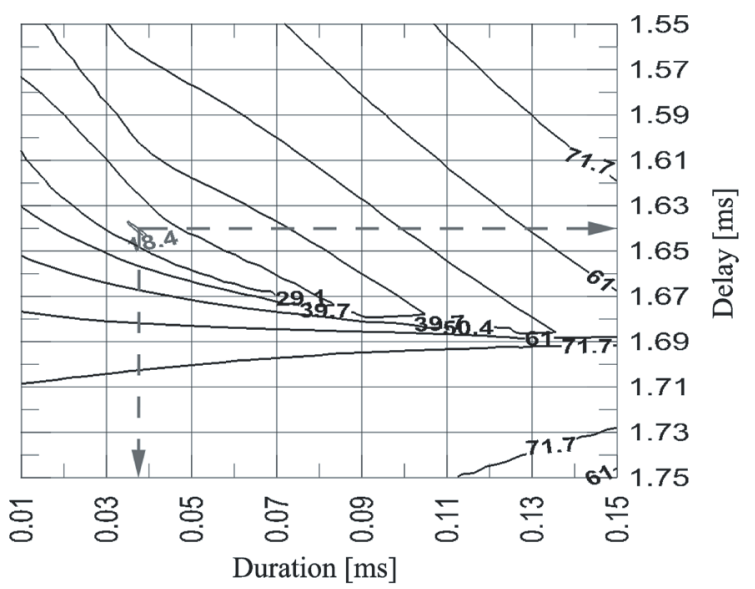

Fig. 14. Contour plot of the opening phase energy [mJ] as function of the two interruption parameters

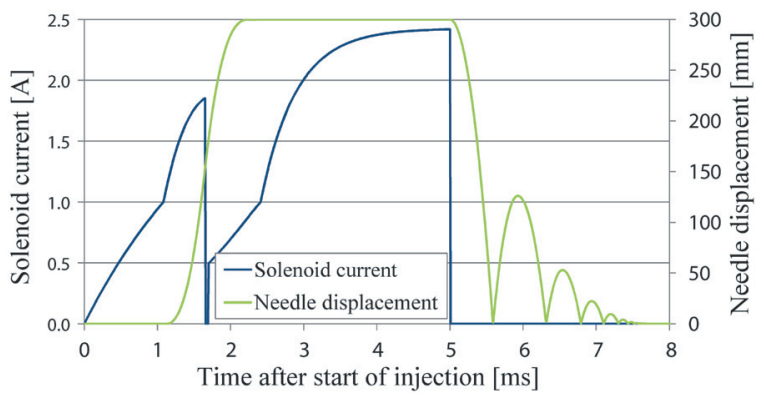

Fig. 15. Model-predicted solenoid current and needle displacement with optimal interruption parameters

As can be seen, the modulation of the injection energy actuated by means of the pulse interruption has the effect of letting the needle reach the open stop surface without impacts and hence without producing bounces; obviously, the best result is attained simply by preventing the first impact from occurring: once the needle rests in the open position, the mass flow remains constant and the injected mass becomes a linear function of the injection time. As a result, Fig. 16 shows the injector flow chart obtained, adopting the optimal interruption parameters for each injection; as is evident, a satisfying linearization has been achieved, since most of the nonlinearities have been suppressed, and the injector characteristic is now a monotone function of the injection time whose deviation from linearity is $\pm 0.22 \mathrm{mg}$, which is significantly lower than the $\pm 1 \mathrm{mg}$ of the original injector flow chart. 
According to these results, a very good linearization of the injector flow chart can be obtained by means of a simple injection pulse modulation; this kind of power supply strategy can be easily implemented in the current production engines by means of a simple ECU software update and without any hardware change. As already mentioned, the suppression of gas injectors' needle bounces have also been studied [9] in order to prevent fatigue stress damages; in this case, however, the proposed method relies on a substantial modification of the injector power supply system.

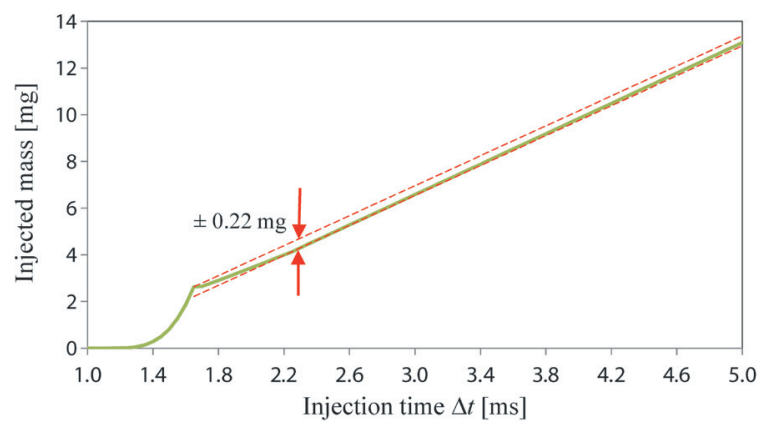

Fig. 16. Simulated optimal injector flow chart ( $\delta=1.64 \mathrm{~ms}, \tau=0.038 \mathrm{~ms}$ )

It is worth mentioning, however, that for a fixed injection time, the injected mass mainly depends on the solenoid current (and hence on the power supply voltage) and on the gas pressure; these two parameters are therefore of crucial importance for the optimization of the injection interruption. With regard to the application in passenger vehicles, the gas pressure can be considered constant, while battery voltage may change during engine operation; the determination of the optimal interruption parameters should therefore be carried out for different supply voltage levels, so as to always adopt the best couple of delay $\delta$ and duration $\tau$.

\section{EXPERIMENTAL VALIDATION}

Once determined through simulations, the optimal injection strategy has been put to the test to experimentally prove its capability to linearize a real injector flow chart.

An experimental campaign has been carried out on a suitably equipped test bench, whose main elements are shown in Fig. 17. The air flow from the cylinder was measured using a Bronkhorst mini CORI-FLOW M13, a Coriolis-type mass flow meter that features a measuring range of 100 to $2000 \mathrm{~g} / \mathrm{h}$ with an accuracy of $\pm 0.2 \%$ of the measured value; after metering, air pressure was reduced to 10 bar before reaching the gas injector, whose activation power was generated by a 13 volt DC power supply. In place of the engine ECU, a National Instruments PCI 6602 counter board programmed with LabVIEW has been employed for the generation of the $0-5$ volt TTL pulses necessary for injector actuation.

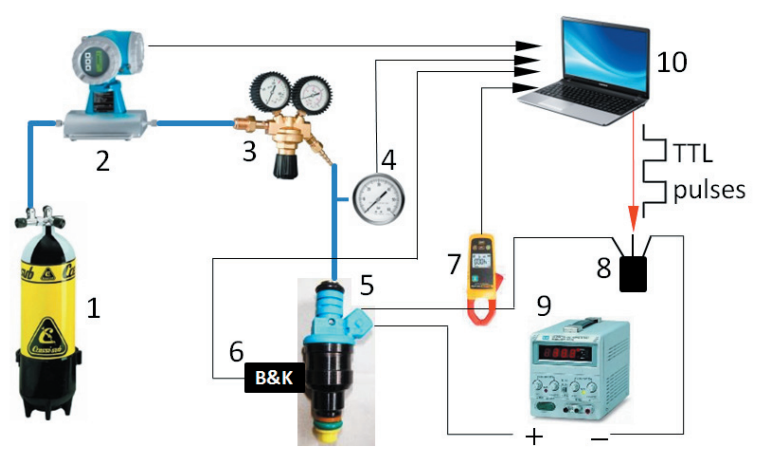

Fig. 17. Injector test bench layout: 1) air cylinder, 2) mass flow meter, 3) pressure regulator, 4) pressure sensor, 5) injector, 6) accelerometer, 7) ammeter, 8) transistor, 9) power supply, 10) signal acquisition and generation system

As also shown in Fig. 3, a transistor was used to transform the low power digital pulses into the high current square waveforms necessary for injector solenoid excitation. The injector was activated with frequencies ranging from 10 to $70 \mathrm{~Hz}$ so as to obtain mass flows in the measurable range; for each injection time, the experimental injected mass $m_{\exp }$ was derived from the measured mass flow $\dot{m}$ and injection frequency $f_{i n j}$ :

$$
m_{\text {exp }}=\frac{\dot{m}}{f_{i n j}} .
$$

The needle impacts were detected via the output signal from a Bruel \& Kjaer Cubic DeltaTron 4502 accelerometer placed on the injector armature, while a clamp-on ammeter LEM PR20 (with 20 $\mathrm{kHz}$ frequency response) was used to acquire the solenoid current. All the necessary quantities have been acquired by means of a National Instruments DAQ board PCI-6133, employing a sample frequency of $400 \mathrm{kHz}$ and using the generated TTL pulse as trigger for data acquisition. For each injection time, the complete waveforms of power supply voltage, solenoid current and accelerometer output were recorded for 100 consecutive injections, while mass flow, gas pressure and temperature were recorded as mean values over the 100 injections. In this way, a complete injector chart could be obtained, e.g. as 
shown in Fig. 6: here the total injected gas mass is reported for each injection time between 1 and $5 \mathrm{~ms}$.

The experimental validation of the optimal injection strategy obviously started from the best pulse interruption parameters determined in the simulation $(\delta=1.64 \mathrm{~ms}, \tau=0.038 \mathrm{~ms})$, even if a certain shift from these values was expected. In effect, although the model replicated with unexpected accuracy the nonlinearities of the injector flow chart, the imperfect correspondence between the experimental results and the model output may, however, cause substantial differences and must be taken into account; for example, the difference in terms of injected mass between model prediction and real measure is shown in Fig. 7, while Fig. 8 shows the phase differences between simulated and real impacts. These differences, even though minuscule, may have a nonnegligible effect on the needle motion and hence on the total injected mass, thus compromising the success of the linearization process. As a consequence, the interruption parameters determined by the model may not represent, as a general rule, the optimal choice even for experimental test. The determination of the best interruption parameters was thus carried out monitoring the output signal from the injector armature accelerometer, searching for the pulse interruption delay $\delta$ and duration $\tau$, which allowed to minimize the needle impacts energy.

The best solution was found for $\delta=1.70 \mathrm{~ms}$ and $\tau=0.1 \mathrm{~ms}$, which are not so far from the model optimal values. In particular, the real best interruption delay $\delta$ was revealed to be very close to the one determined by simulation, while the interruption duration $\tau$ instead showed a greater difference. This can be easily explained taking into account the delay sequence in the injection pulse actuation: the insulated gate bipolar transistor (IGBT) employed in the test, in effect, is characterized by typical current falling and rising times on the order of some tens of microsecond, which indeed delays the needle actuation and contributes to extending the total interruption duration. The result of the experimental linearization achieved is shown in Fig. 18: as can be seen, the linearity of the optimized injector flow chart is not as good as the one obtained by simulation, since the real optimal flow chart revealed a $\pm 0.35 \mathrm{mg}$ deviation from the ordinary least square line, which is higher than the $\pm 0.22 \mathrm{mg}$ of the simulated optimal flow chart of Fig. 16.

The reason for this higher deviation from linearity can be found in the natural dispersion of the measured injected mass around the mean value: as shown in Fig. 19, the experimental measurement dispersion, evaluated by means of the standard deviation recorded for each of the 100 consecutive mass flow samples acquired during the test, can be as high as $0.59 \mathrm{mg}$. This means that, for example, for the fixed injection time of $1.85 \mathrm{~ms}$, even if the mean injected mass is $2.73 \mathrm{mg}$, as reported in the diagrams in Fig. 6 and Fig. 7, the measured 100 consecutive values used to compute this mean are scattered in a $0.59 \mathrm{mg}$ wide range. It should be mentioned that this data dispersion is not caused by mass flow measurement errors, which (as already stated) are less than $1 \%$ of the measured value. The cause of these high dispersions is instead related to the real needle movement, which, even at fixed injection times, does not repeat identically at each injection, thus causing significant variations on the total injected mass.

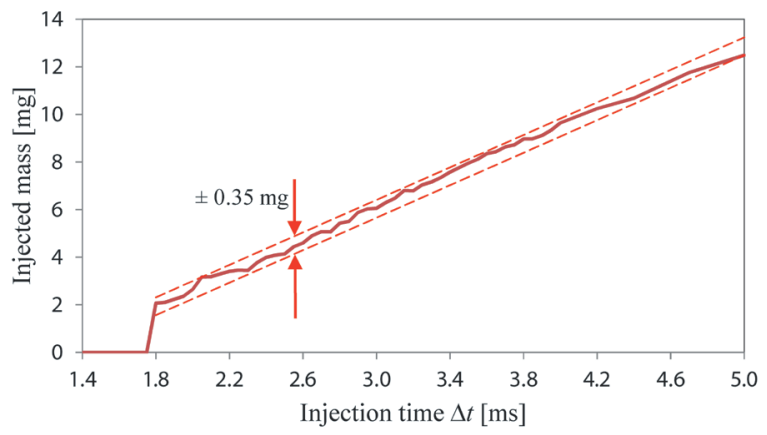

Fig. 18. Experimental optimal injector flow chart $(\delta=1.70 \mathrm{~ms}, \tau=0.10 \mathrm{~ms})$

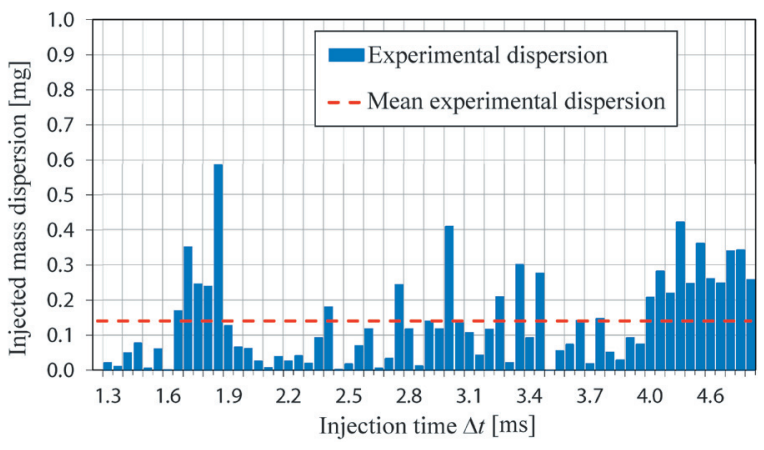

Fig. 19. Dispersion range of the measured injected mass

In conclusion, the result obtained by the simple pulse interruption strategy allowed a substantial improvement of the injector flow chart, whose deviation from linearity has been reduced to about one third of the original chart of Fig. 6. With the aim of evaluating the benefit introduced by the proposed injection strategy on the control of the engine airfuel ratio, the deviation from the OLS lines has been determined for the original injector flow map and for both the simulated and experimental optimized charts; as shown in Fig. 20, the use of an OLS regression 
line in place of the original injector flow chart would cause an air-fuel ratio error up to $37 \%$ in the lower injection time zone; once optimized by means of pulse interruption strategy, the improved linearity of the injector flow chart allows using the OLS line with a maximum error of $10 \%$ in the same lower injection time region: the optimization performed hence may noticeably improve the engine air-fuel ratio control for the lower injection time.

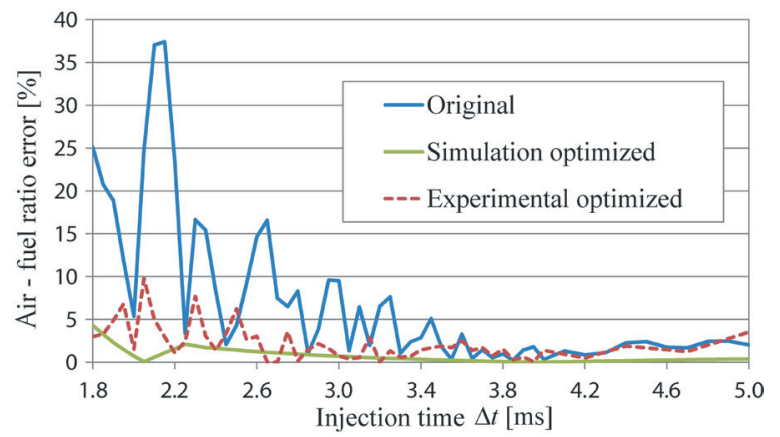

Fig. 20. Air-fuel ratio error caused by the use of the OLS line in place of injector flow map

\section{CONCLUSIONS}

A mathematical model previously realized by the authors for the simulation of the needle motion of a natural gas injector and for the evaluation of the injected mass has been now employed to determine an optimal injection strategy with the aim of linearizing to the greatest extent the nonlinear part of a real S.I. engine gas injector. The analysis of the needle motion, together with some considerations on energy conservation, led to the definition of a proper objective function, which guided the authors toward one possible solution, whose main advantage is to be easily implementable in current engine ECU without any hardware modification or additional costs: it consists of the modulation of the energy transferred to the needle by means of an injection pulse interruption in order to avoid any bounces. The pulse interruption strategy was implemented in the mathematical model, thus allowing the effective linearization of the simulated injector flow chart.

On the basis of the good results obtained with simulations, the authors proceeded to the validation of the solution found by means of experimental tests carried out on a suitably equipped test bench. A real injector was controlled with the pulse interruption strategy, adopting as initial parameters the values suggested by simulation. The best interruption parameters were experimentally fixed, and allowed to substantially improve the injector flow chart, whose deviation from linearity was reduced to one third of the original flow chart: the authors consider this result to be undeniably good, especially if the measurement dispersion of the injected mass is considered. As outlined, power supply voltage and gas pressure are of crucial importance for the optimization of the injection interruption. For application in passenger vehicles, gas injection pressure can be considered constant, while battery voltage variations may occur; to take this into account, the determination of the optimal interruption parameters should be carried out for different supply voltage levels.

\section{NOMENCLATURE}

C.I. Compression Ignition

CNG Compressed Natural Gas

ECU Electronic Control Unit

IGBT Insulated Gate Bipolar Transistor

LPG Liquefied Petroleum Gas

MPC Model Predictive Control

OLS Ordinary Least Square

S.I. Spark Ignition

TTL Transistor to Transistor Logic

$E \quad$ energy transferred to the injector needle during the opening phase $[\mathrm{mJ}]$

$E_{\text {coil }} \quad$ energy stored in the solenoid coil [mJ]

$f_{i n j} \quad$ injection frequency $[\mathrm{Hz}]$

$i \quad$ solenoid current [A]

$L \quad$ solenoid inductance $[\mathrm{mH}]$

$m_{\text {exp }} \quad$ experimental injected mass $[\mathrm{mg}]$

$\dot{m} \quad$ mass flow $[\mathrm{g} / \mathrm{s}]$

$R \quad$ equivalent resistance $[\Omega]$

$t \quad$ time [ms]

$t_{0} \quad$ time at the end of the rapid discharge

phase [ms]

$V \quad$ voltage [V]

$V_{0} \quad$ voltage at the end of the rapid discharge phase [V]

$V_{1} \quad$ asymptotic voltage of the discharge phase [V]

$\delta \quad$ time delay of the injection interruption [ms]

$\phi \quad$ objective function of the optimal condition search algorithm $[\mathrm{mJ}]$

$\tau \quad$ duration of the injection interruption [ms]

$\Delta t \quad$ injection time [ms]

$t^{*} \quad$ opening phase duration [ms]

$A \quad$ solenoid cross-section area $\left[\mathrm{mm}^{2}\right]$

$B \quad$ magnetic induction in the steel [T]

$B_{0} \quad$ magnetic induction in the air [T]

c viscous damping coefficient $[\mathrm{N} /(\mathrm{m} / \mathrm{s})]$

$F_{a m b} \quad$ ambient pressure force $[\mathrm{N}]$ 
$F_{\text {em }} \quad$ electromagnetic force [N]

$F_{f r} \quad$ Coulomb frictional force $[\mathrm{N}]$

$F_{\text {gas }} \quad$ gas pressure force [N]

$F_{v} \quad$ gas viscous force [N]

$H \quad$ magnetic field in the steel $[\mathrm{A} / \mathrm{m}]$

$H_{0} \quad$ magnetic field in the air $[\mathrm{A} / \mathrm{m}]$

$i \quad$ solenoid coils current [A]

$k \quad$ spring constant $[\mathrm{N} / \mathrm{mm}]$

$M \quad$ needle mass [g]

$N \quad$ number of coils enclosed by the loop

$R \quad$ solenoid electric resistance $[\Omega]$

$V \quad$ injector power supply voltage [V]

$x \quad$ needle position [mm]

$\dot{x} \quad$ needle velocity $[\mathrm{m} / \mathrm{s}]$

$\dot{x}_{a i} \quad$ needle velocity after impact $[\mathrm{m} / \mathrm{s}]$

$\dot{x}_{b i} \quad$ needle velocity before impact [m/s]

$\ddot{x} \quad$ needle acceleration $\left[\mathrm{mm} / \mathrm{ms}^{2}\right]$

$\delta_{S} \quad$ spring preload deformation [mm]

$\phi_{B} \quad$ magnetic induction flux [Wb]

$\mu_{0} \quad$ space magnetic permeability $[\mathrm{H} / \mathrm{m}]$

$\mu_{r} \quad$ steel relative magnetic permeability $[\mathrm{H} / \mathrm{m}]$

$\sigma \quad$ path along the loop [mm]

$\xi \quad$ coefficient of restitution [-]

\section{REFERENCES}

[1] Cammalleri, M., Pipitone, E., Beccari, S., Genchi, G. (2013). A mathematical model for the prediction of the injected mass diagram of a S.I. engine gas injector. Journal of Mechanical Science and Technology, vol. 27, no. 11, p. 3253-3265, DOI:10.1007/s12206-0130848-6.

[2] Lino, P., Maione, B., Rizzo, A. (2007). Nonlinear modelling and control of a common rail injection system for diesel engines. Applied Mathematical Modelling, vol. 31, no. 9, p. 1770-1784, DOI:10.1016/j. apm.2006.06.001.

[3] Seykens, X.L.J., Somers, L.M.T., Baert, R.S.G. (2005). Detailed modelling of common rail fuel injection process. Journal of Middle European Construction and Design of Cars, vol. 3, no. 2-3, p. 30.

[4] Le, D., Shen, J., Ruikar, N., Shaver, G.M. (2013). Dynamic modeling of piezoelectric fuel injector during rate shaping. International Journal of Engine Research, vol. 15, no. 4, p. 471-487, DOI:10.1177/1468087413492737.

[5] Mehlfeldt, D., Weckenmann,H., Stohr, G. (2008). Modeling of piezoelectrically actuated fuel injectors. Mechatronics, vol. 18, no. 5-6, p. 264-272, DOI:10.1016/j.mechatronics.2008.03.001.

[6] Baratta, M., Catania, A.E., Spessa, E., Herrmann, L., Roessler, K. (2009). Multi-dimensional modeling of direct natural-gas injection and mixture formation in a stratified-charge SI engine with centrally mounted injector. SAE International Journal of Engines, vol. 1, no. 1, p. 607-626, DOI:10.4271/2008-01-0975.
[7] Di Cairano, S., Bemporad, A., Kolmanovsky, I.V., Hrovat, D. (2007). Model predictive control of magnetically actuated mass spring dampers for automotive applications. International Journal of Control, vol. 80, no. 11, p. 1701-1716, DOI:10.1080/00207170701379804.

[8] Lino, P., Maione, B., Amorese, C., De Matthaeis, S. (2008). Modeling and predictive control of a new injection system for compressed natural gas engines. Control Engineering Practice, vol. 16, no. 10, p. 12161230, DOI:10.1016/j.conengprac.2008.01.008.

[9] Dyntar, D., Guzzella, L. (2004). Optimal control for bouncing suppression of $\mathrm{CNG}$ injectors. Journal of Dynamic Systems, Measurement and Control (ASME), vol. 126, no. 1, p. 47-53, DOI:10.1115/1.1648311.

[10] Bosch Automotive Handbook (1996). Robert Bosch GmbH, Bentley Publishers, Cambridge, from: http:// www.bentleypublishers.com/c/H016, accessed on 201403-25.

[11] Pipitone, E., Beccari, S. (2009). Performances improvement of a S.I. CNG bi-fuel engine by means of double-fuel injection. SAE International, Technical Paper 2009-24-0058, DOI:10.4271/2009-24-0058.

[12] Pipitone, E., Beccari, S. (2010). Performance and emission improvement of a S.I. engine fuelled by LPG/ gasoline mixtures. SAE International, Technical Paper 2010-01-0615, DOI:10.4271/2010-01-0615.

[13] Obiols, J., Soleri, D., Dioc, N., Moreau, M. (2011). Potential of concomitant injection of $\mathrm{CNG}$ and gasoline on a $1.6 \mathrm{~L}$ Gasoline Direct Injection Turbocharged Engine. SAE International, Technical Paper 2011-011995, DOI:10.4271/2011-01-1995.

[14] Momeni Movahed, M., Basirat Tabrizi, H., Mirsalim, M. (2014). Experimental investigation of the concomitant injection of gasoline and $\mathrm{CNG}$ in a turbocharged spark ignition engine. Energy Conversion and Management, vol. 80, p. 126-136, DOI:10.1016/j. enconman.2014.01.017.

[15] Veiga, M., Mansano, R., Silva, R., Gomes, C. (2010). Injection system for tri-fuel engines with control of power by simultaneous use of $\mathrm{CNG}$ and ethanol or gasoline, SAE International, Technical Paper 2010-360195, DOI:10.4271/2010-36-0195.

[16] Beccari, S., Pipitone, E., Cammalleri, M., Genchi, G. (2014). Model-based optimization of injection strategies for SI engine gas injectors. Journal of Mechanical Science and Technology, vol. 28, no. 8, p. 1-13, DOI:10.1007/s12206-014-0742-x.

[17] Fairchild Semiconductor Corporation, "HGTP14N40F3VL/HGT1S14N40F3VLS Data sheet Rev. B1", February 2002, http://www.datasheetlib. com/datasheet/184055/hgtp14n40f3vl_fairchildsemiconductor.html, accessed on 2014-03-25.

\section{APPENDIX A}

As already mentioned, the first tests carried out interrupting the injection pulse with the aim of 
modulating the energy transferred to the needle showed a phenomenon not observed in the previous work: the experimental data in effect revealed an extra-current whose duration and magnitude depends on the duration of the pulse interruption, as shown in Fig. 21. Here, different solenoid current waveforms, obtained by varying the pulse interruption duration, are represented as functions of time: as can be seen, the extra-current amplitude decreases when the interruption duration increases.

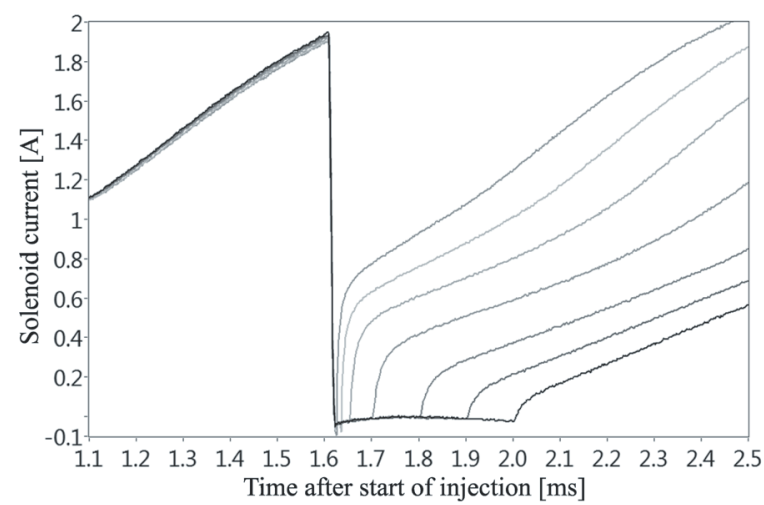

Fig. 21. Measured solenoid current for different duration of the pulse interruption

The analysis of the injection electric circuit (here reported in Fig. 22) and of the IGBT characteristics [17], together with some voltage measurements (carried out between points A and B of the electric circuit) led the authors to believe that the phenomenon is related to the dissipative discharge of the energy accumulated in the solenoid coil.

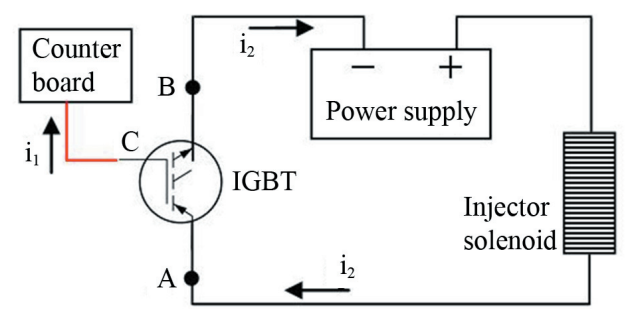

Fig. 22. Electrical circuit involved in the injector operation

When the injection is interrupted, the IGBT is deactivated, and this abruptly opens the electric circuit (between points A and B); the solenoid current immediately falls down to zero, and this causes an abrupt decrease of the solenoid magnetic flux, which, according to the Faraday-Lenz law, induces a very high voltage in the solenoid. A waveform of this high voltage has been recorded by means of a $100 \mathrm{MHz}$ oscilloscope and is reported in Fig. 23.
As can be seen, the voltage induced in the solenoid circuit exceeds $390 \mathrm{~V}$ (which is also the oscilloscope maximum visible value): as a consequence, the IGBT intrinsic protection system, endowed of Zener diodes and internal resistances, permits this high voltage to discharge through itself toward the ground of the counter board used to generate the digital pulses (current $i_{1}$ in Fig. 22). This first part of the solenoid energy discharge is very rapid, as can be noted in Fig. 23.

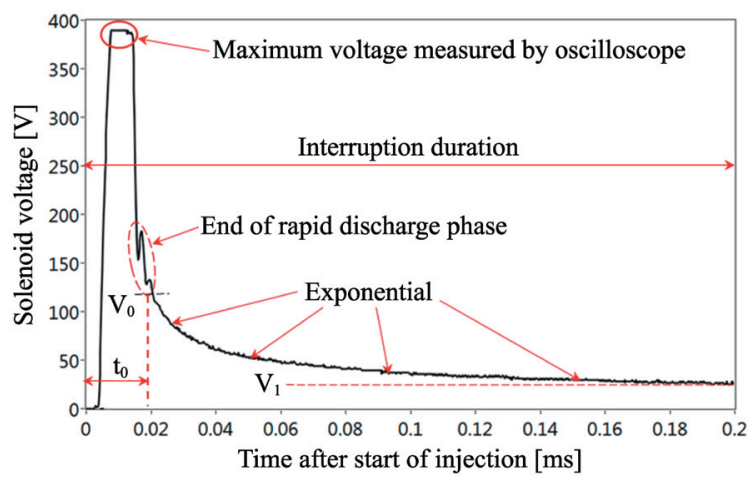

Fig. 23. Measured solenoid voltage during the injection pulse interruption

Once below $130 \mathrm{~V}$ (approximately $0.02 \mathrm{~ms}$ after the start of pulse interruption), due to the IGBT intrinsic protection system properties [17], the path to the counter board ground through the IGBT closes; therefore, the energy continues to discharge through the internal structure of the injector itself; this second stage of the discharge process, as can be observed in Fig. 23, is slower, and the voltage exhibits a gradual decrease. Considering a simple R-L circuit, this voltage waveform has been fitted by an exponential function of time $t$ :

$$
V=V_{1}+\left(V_{0}-V_{1}\right) \cdot e^{-\left(t-t_{0}\right) \cdot \frac{R}{L}}
$$

where $t_{0}$ and $V_{0}$ represent the time and the voltage at the end of the rapid discharge phase $(0.02 \mathrm{~ms}$ and 130 $V$ respectively), $V_{1}$ is the asymptotic voltage (fixed by the power supply system) and $L / R$ is the time constant of the circuit: in particular $L$ is the known solenoid inductance while $R$ is the equivalent resistance, which has been determined fitting the data of Fig. 23 with Eq. (3).

The instantaneous energy stored in the solenoid can be always expressed as:

$$
E_{\text {Coil }}=\frac{1}{2} \cdot L \cdot i(t)^{2}
$$


where, according to Eq. (3), the current $i(t)$ during the slow discharge phase can be evaluated as:

$$
i=\frac{\left(V_{0}-V_{1}\right)}{R} \cdot e^{-\left(t-t_{0}\right) \cdot \frac{R}{L}} .
$$

If, during this slow discharge phase, after a sufficiently short time (less than $0.2 \mathrm{~ms}$ from the start of pulse interruption) the electric circuit is closed again (i.e. the IGBT is reactivated), the residual energy still stored in the solenoid suddenly discharges through the power supply cathode, producing the extra current $\left(i_{2}\right.$ in Fig. 22), whose value depends on the energy still available in the solenoid, and can be evaluated by Eq. (5); the value of this extra current is therefore related to the duration of the interruption itself: this explains the current waveforms represented in Fig. 21.

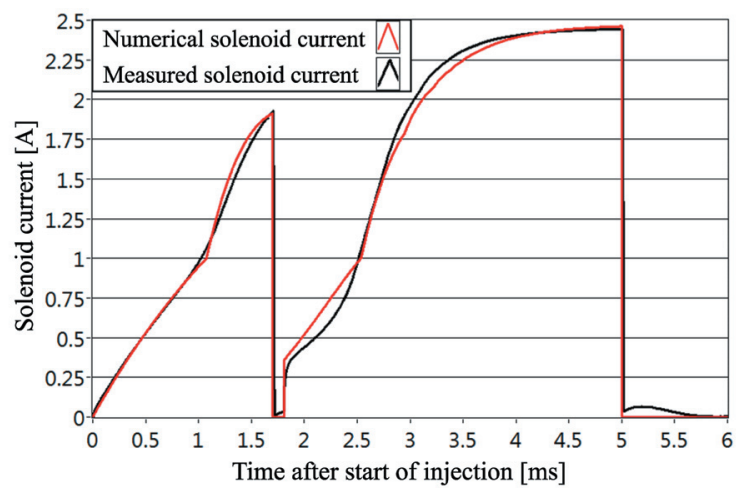

Fig. 24. Injection pulse interruption: measured and simulated solenoid current for air at 10 bar

Eqs. (3) and (5) have been implemented in the model with the aim to take account of the extra current phenomenon: Fig. 24 shows the good agreement between experimental measure and simulation output.

\section{APPENDIX B}

The mathematical model realized by the authors [1] is able to predict the needle motion during an entire injection event and to evaluate the total injected mass for any fixed operative condition. A brief description of the main physical principles taken into consideration to realize the mathematical model follows.

From the electrical point of view, the injector has been modelled as an electromagnet, schematically represented in Fig. 25.

When the current $i$ flows through the injector solenoid coils, a magnetic field $H$ appears in the steel core according to the Ampère's circuital law, which correlates the integrated magnetic field around a closed loop to the electric current flowing through the loop.

$$
\oint H \cdot d \sigma=N \cdot i,
$$

where $N$ is the number of coils enclosed by the loop.

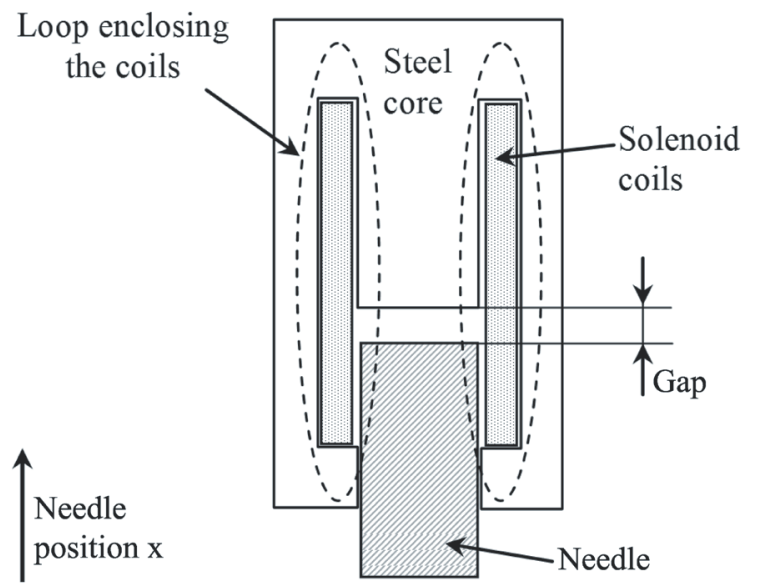

Fig. 25. Schematic representation of the injector magnetic circuit

While the magnetic $H$-field changes passing from the steel to the air gap, the magnetic induction $B$ does not change, hence:

$$
B_{0}=B \text {, }
$$

where subscript 0 refers to the air gap.

$B$ and $H$ fields are connected by the constitutive equation:

$$
B=\mu_{0} \cdot \mu_{r} \cdot H
$$

being $\mu_{0}$ the space permeability and $\mu_{r}$ the steel relative permeability (function of $H$ ).

Combining the above equations allows definition of the relation $B=B(i, x)$ between the magnetic $B$-field, the solenoid current $i$ and the needle position $x$. Furthermore, Ohm's law together with FaradayLenz's law yields:

$$
R \cdot i=V-\frac{d \varphi_{B}}{d t},
$$

where $R$ represents the solenoid electric resistance, $\phi_{B}=N \cdot B \cdot A=\phi_{B}(i, x)$ the flux of the magnetic $B$-field and $A$ is the solenoid cross-section area; in this way, the solenoid current is connected to its time derivative and to the needle motion.

The relation between the electromagnetic force $F_{e m}$ and the solenoid current $i$ can be easily determined considering that for any virtual displacement $d x$ of 
the needle, the work produced by $F_{e m}$ must equal the electromagnetic energy variation in the gap:

$$
F_{e m} \cdot d x=\frac{B_{0}^{2}}{2 \cdot \mu_{0}} \cdot S \cdot d x=\frac{[B(i, x)]^{2}}{2 \cdot \mu_{0}} \cdot S \cdot d x,
$$

being

$$
\int_{0}^{H} H \cdot d B=\frac{B_{0}^{2}}{2 \cdot \mu_{0}}
$$

the magnetic energy per unit volume in the gap.

From the mechanical point of view, the injector has been modelled as a mass-spring system, damped by Coulomb and viscous frictional forces, and subject to variable electromagnetic and constant gas pressure forces. Based on the free body diagram of Fig. 26, the needle dynamic equilibrium equation can be written as:

$$
\begin{gathered}
M \cdot \ddot{x}+c \cdot \dot{x}+k \cdot x= \\
=F_{e m}-k \cdot \delta_{S}-F_{f r} \frac{\dot{x}}{|\dot{x}|}-\left(F_{g a s}-F_{a m b}\right),
\end{gathered}
$$

where $M$ represents the needle mass, $c$ the viscous damping coefficient, $k$ and $\delta_{S}$ the spring constant and preload deformation, respectively; $F_{f r}$ is the coulomb frictional force, $F_{\text {gas }}$ is the force exerted by the gas pressure while $F_{a m b}$ is the ambient pressure force. This equation correlates the needle position $x$ to its first and second time derivative and to the solenoid current $i$.

Finally, to take in to account the needle impacts and bounces on the seat surfaces, the following equation has been introduced:

$$
\dot{x}_{a i}=-\sqrt{\xi} \cdot \dot{x}_{b i}
$$

where $\dot{x}_{a i}$ and $\dot{x}_{b i}$ are the needle velocities after and before impact respectively, and $\xi$ is the coefficient of restitution (i.e. the ratio between the kinetic energy after and before impact).

In summary, in the mathematical model realized, both solenoid current and needle motion are fully described by the two coupled differential equations (Eqs. (9) and (12)), together with the Eq. (13) applied at each impact.

Assuming the injector equivalent to a choked flow convergent nozzle, the total injected mass results proportional to the value of the integral of the needle displacement over time; this allows determination of the total amount of fuel supplied at the end of each injection event.

The above physical equations were then expressed in a dimensionless form [1], thus strongly reducing the number of parameters required for model calibration.

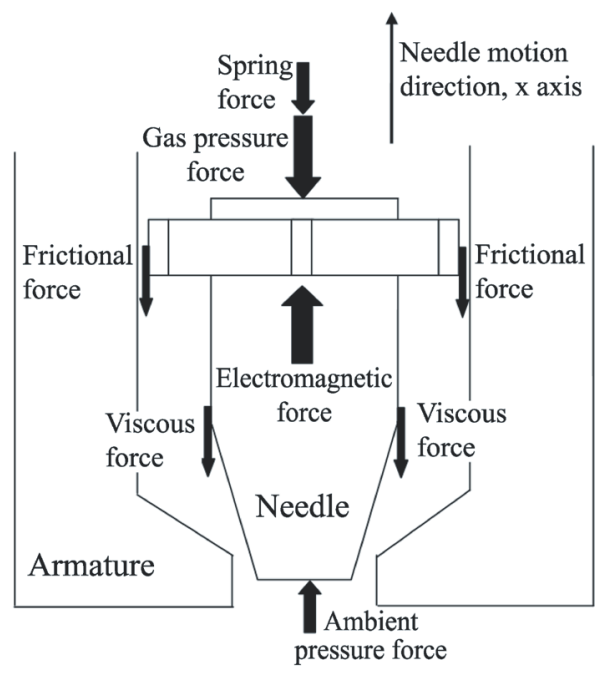

Fig. 26. Injector needle free body diagram 